\title{
The mapping of visual space by identified large second-order neurons in the dragonfly median ocellus
}

\author{
Richard Berry · Gert Stange $\cdot$ Robert Olberg • \\ Joshua van Kleef
}

Received: 2 February 2006/Revised: 9 May 2006/ Accepted: 14 May 2006/Published online: 8 June 2006

(C) Springer-Verlag 2006

\begin{abstract}
In adult dragonflies, the compound eyes are augmented by three simple eyes known as the dorsal ocelli. The outputs of ocellar photoreceptors converge on relatively few second-order neurons with large axonal diameters (L-neurons). We determine L-neuron morphology by iontophoretic dye injection combined with three-dimensional reconstructions. Using intracellular recording and white noise analysis, we also determine the physiological receptive fields of the Lneurons, in order to identify the extent to which they preserve spatial information. We find a total of 11 median ocellar L-neurons, consisting of five symmetrical pairs and one unpaired neuron. L-neurons are distinguishable by the extent and location of their terminations within the ocellar plexus and brain. In the horizontal dimension, L-neurons project to different regions of the ocellar plexus, in close correlation with their receptive fields. In the vertical dimension, dendritic arborizations overlap widely, paralleled by receptive fields that are narrow and do not differ between different neurons. These results provide the first evidence for the preservation of spatial information by the second-order neurons of any dorsal ocellus. The system essentially forms a one-dimensional image of the equator over a wide azimuthal area, possibly
\end{abstract}

R. Berry $(\bowtie) \cdot$ G. Stange $\cdot$ J. van Kleef

Centre for Visual Sciences, Research School of Biological

Sciences, Australian National University, PO Box 475,

Canberra, ACT 2601, Australia

e-mail: rberry@rsbs.anu.edu.au

R. Olberg

Biological Sciences, Union College,

Schenectady, NY, USA forming an internal representation of the horizon. Potential behavioural roles for the system are discussed.

Keywords Dragonfly · Ocelli $\cdot$ L-neuron · White noise $\cdot$ Receptive field $\cdot$ Three-dimensional reconstructions
Abbreviations
L-neuron Large second-order ocellar neuron
LED Light emitting diode
PSL Posterior Slope
S-neuron Small second-order ocellar neuron UV Ultraviolet

\section{Introduction}

A common feature of both the single lens eyes of vertebrates, and the compound eyes of arthropods, is the presence of imaging optics, followed by multiple layers of neurons. Typically, a photoreceptor layer is followed by several layers of interneurons, such as the horizontal, bipolar and amacrine cells in vertebrates, or the lamina and medulla neurons in arthropods. A layered organization that preserves the spatial relationship between subsequent layers is one of the prerequisites to maintain the spatial mapping of visual space. Another prerequisite is the presence of interneurons in numbers that are sufficient to maintain spatial mapping, and it is found, indeed, that pronounced convergence tends to be lacking at the periphery. 
This does not apply to the dorsal ocelli as found in many adult insects. Present in parallel to the compound eyes, the ocelli are simple, single lens eyes that typically occur as a triplet on the head of flying insects. It has been frequently demonstrated that the refracting power of ocellar lenses is not sufficient to form a focused image on the retina (Homann 1924; Parry 1947; Cornwell 1955; Wilson 1978). While the ocellar retina may contain thousands of receptor neurons, the outputs of these neurons converge in the ocellar neuropile onto only a few tens of second-order neurons. Areas of integration between photoreceptors and higher-order neurons are not easily defined, and it has been shown in bees (Guy et al. 1979) and cockroaches (Toh et al. 1983) that the ocellar tract itself contains synaptic areas along its length. Therefore there is little, if any, evidence for the presence of either layering or horizontal connections via separate interneurons.

Underfocussed dioptrics in combination with pronounced convergence ratios suggest that the ocellar system is not suitable for spatial mapping of the visual environment. Alternative functions for the ocelli must therefore be considered. Wilson (1978) proposed that the ocelli are designed to detect global changes in light intensity over a large area of visual space. During flight, deviations about the pitch or roll axes are encoded by comparing relative changes in the intensity measured by each of the three ocelli. The output from the ocelli is then fed into wing muscle control systems to provide a rapid correction system for deviations in pitch or roll. Wehner (1987) describes this system as a 'matched filter' for horizon detection where both speed and sensitivity have been optimised while spatial resolution (which is undesirable) has been eliminated.

Typically, the second-order neurons of the ocelli are divided into two categories: a small number of large second-order neurons (L-neurons) with axon diameters exceeding $8 \mu \mathrm{m}$, and the more numerous small neurons (S-neurons) with axon diameters of $5 \mu \mathrm{m}$ or less (Goodman 1981). Of these two categories, three attributes of the L-neurons suggest that they are particularly suitable for the rapid correction of body attitude during flight. Firstly, the sheer size of the L-neurons, which in the dragonfly may reach diameters of up to $30 \mu \mathrm{m}$ (Chappell et al. 1978), marks them as a prominent feature of the insect brain, and suggests that they are optimised for high transmission velocity. Secondly, L-neurons may either directly innervate wing motor ganglia, or do so via a small number of interneurons (Strausfeld 1976; Guy et al. 1979; reviewed in Mizunami 1994). Lastly, L-neurons have wide dendritic arborizations in the ocellar plexus (Goodman 1976), which argues for correspondingly wide receptive fields.
Wilson (1978) determined angular sensitivity functions from L-neurons in locust lateral ocelli, along a horizontal trajectory, and found mean acceptance angles of $80^{\circ}$. Wide receptive fields further increase sensitivity at the expense of resolution.

While this notion remains unchallenged for most insect ocellar systems, there is a mounting body of evidence indicating that it does not apply in the case of dragonflies. The dragonfly median ocellus is capable of considerable resolving power because the lens is unusually thick and strongly curved, and the ultrastructure suggests adaptation for resolution (Stange et al. 2002). By observing reflected light from the tapetal layer in the retina of Hemicordulia tau, the latter authors found that an incident beam of parallel light is focused onto a patch that extends over angles of $12^{\circ}$ in elevation and $24^{\circ}$ in azimuth. A direct proof that spatial information is preserved at the level of the retina was provided by electrophysiological evidence from photoreceptor neurons, showing that individual photoreceptors have mean acceptance angles of $15^{\circ}$ in elevation and $28^{\circ}$ in azimuth (van Kleef et al. 2005).

Consequently, the dragonfly median ocellus forms a spatial map of the visual environment up to the output of the receptor neuron layer, immediately raising the question as to what extent this mapping is preserved in the second-order neurons. The present study addresses this question by combining both structure and function. We start with the anatomy of the dragonfly median ocellar L-neurons, supplementing earlier work by expanding and completing previous knowledge, including complete reconstructions that image the afferent projections in two dimensions. We then combine this with electrophysiological determination of the sizes, shapes and locations of spatial receptive fields, also in two dimensions. This undertaking would not have been possible without the availability of relatively novel utilities, namely confocal microscopy for the purpose of anatomical three-dimensional reconstructions, and white noise analysis for the purpose of reconstructing physiological receptive fields.

\section{Materials and methods}

\section{Experimental animals}

All recordings and histology were performed on adult dragonflies of the species Hemicordulia tau or Hemicordulia australiae. Dragonflies were either caught locally in the area of Canberra, Australia or reared to adulthood from larvae. Larvae were induced into emergence by relocation to a tank filled with warm 
$\left(20^{\circ} \mathrm{C}\right)$ water that was made slightly saline by adding an artificial sea salt mixture (Instant Ocean, $2 \mathrm{~g} / \mathrm{l}$ ), and regular feeding with blackworms (Lumbriculus) or water fleas (Daphnia). Adult dragonflies were typically stored in the dark at $4^{\circ} \mathrm{C}$ for a period of 1-3 days after capture or emergence before being used for electrophysiology.

\section{Preparation}

Dragonflies were secured by their dorsal surface to a wax-covered rod, $6 \mathrm{~mm}$ in diameter, by gently wrapping the wings around the rod and pressing them into the wax. The dragonfly was oriented with the ventral surface facing upwards and tilted at an angle of $45^{\circ}$ for ease of dissection and electrode penetration. A perspex plate with a narrow slot to accommodate the neck was slipped between the head and prothorax. The caudal rims of the compound eyes were then secured to the plate with Periphery wax (Surgident). The legs of the dragonfly were immobilised by wrapping a piece of insulation tape around the thorax. The median ocellar nerve and ventral aspect of the brain were exposed by making a horizontal incision just anterior to the compound eyes to remove the frons and labrum. Air sacs overlying the ventral aspect of the brain were gently removed and the entire brain was bathed in dragonfly saline $(105 \mathrm{mM} \mathrm{NaCl}, 1.5 \mathrm{mM} \mathrm{KCl}, 0.9 \mathrm{mM} \mathrm{CaCl}$, $2.4 \mathrm{mM} \mathrm{NaHCO} 3$ ). The mandibles were either removed or waxed together to minimise movement within the head capsule. Occasionally the dragonfly was mounted in an inverted position whereby the ventral side of the thorax was waxed to the stand (after removing the legs). This allowed manipulation and observation of the wings while simultaneously recording from the L-neurons. However, leaving the wings free in this fashion reduced the stability of recordings obtained from these preparations. All recordings were obtained from the median ocellar nerve.

\section{Intracellular recording}

The median ocellar nerve was supported from below by positioning a steel spoon underneath the dorsal surface of the nerve adjacent to the brain. A silver chloride wire, used as the indifferent electrode, was either placed into the compound eye or inserted directly adjacent to the median ocellar nerve. Microelectrodes were pulled by a P-87 Flaming/Brown Micropipette puller from thin walled $1.0-0.78 \mathrm{~mm}$ borosilicate class (SDR Clinical Technologies). Electrode tips were filled by capillary action with $3-10 \%$ Lucifer Yellow $\mathrm{CH}$ (Sigma Chemicals) in $1 \% \mathrm{LiCl}$ solution and then backfilled with $1 \mathrm{M} \mathrm{LiCl}$. To allow identification of multiple neurons from a single preparation some cells were labelled with Rhodamine Dextran instead of Lucifer Yellow. In these cases the electrode tip was filled with 3\% Tetramethylrhodamine Dextran (Molecular Probes) in $0.5 \mathrm{M}$ potassium acetate and backfilled with $0.5 \mathrm{M}$ potassium acetate. Lucifer Yellow was preferred over Rhodamine Dextran as the latter had a tendency to leak out of the cell. In either case, electrodes typically had a DC resistance of 40-60 M $\Omega$. The output from the electrode was passed through a Getting 5A preamplifier, observed on an oscilloscope and recorded on computer.

Electrodes were fastened to a Leitz micromanipulator and advanced through the ventral surface of the median ocellar nerve until a sudden drop in voltage of 40-50 mV was encountered. The electrode was considered to be intracellular if the response to a sudden flash of light was similar to those of dragonfly L-neurons described previously (Chappell and Dowling 1972; Patterson and Chappell 1980; Mobbs et al. 1981).

\section{Stimuli}

Two types of visual stimuli were used to analyse the physiological properties of the L-neurons. Initially a point light source was used, composed of one green (Roithner Lasertechnik B5-433-B525, peak emission at $528 \mathrm{~nm}$ ) and one UV LED (Roithner Lasertechnik 380D15, peak emission at $383 \mathrm{~nm}$ ), which matches the spectral sensitivity of dragonfly ocellar photoreceptors (Chappell and DeVoe 1975). LEDs were attached to a cardan arm, which allowed stepwise rotation in both elevation and azimuth around the head of the dragonfly, such that the stimulus could be moved to any point within the visual field of the median ocellus (Stange et al. 2002). Rotation around the head was controlled by means of a stepper motor that was under direct control of a computer running a custom written $\mathrm{C}$ program. The program also allowed simultaneous control of stimulus intensity, over a $2 \log$ unit range, while recording responses from the neuron. In spite of its versatility, this stimulus was found to be inadequate for the characterisation of the receptive fields of the Lneurons (see Results section). Consequently, it was necessary to develop a wide field green/UV stimulus and combine it with a white noise analysis whereby the contrast levels at all spatial locations are simultaneously modulated. This combination circumvents the problem of adaptation and allows a very rapid and complete analysis of the receptive fields (Sakai et al. 1988). 
The wide field stimulus is an advanced version of that previously used in our laboratory to analyse the spatiotemporal properties of dragonfly ocellar photoreceptors (van Kleef et al. 2005). The original stimulus consisted of a single column of green and UV LEDs. The version described here was extended to cover two dimensions by creating 9 columns of LEDs with each column containing 12 side-by-side pairs of green and UV LEDs of the same make as above (Fig. 1). The stimulus thus consisted of a total of 216 LEDs arranged on the surface of a sphere of radius $120 \mathrm{~mm}$. The head of the dragonfly was positioned at the centre of the sphere by locating it at the point of intersection of two aligned laser pointers attached to the stimulus. From the point of view of the dragonfly, the intervals between LEDs in the vertical direction (elevation) are $6^{\circ}$, while the intervals in the horizontal direction (azimuth) are $12^{\circ}$ (from the nearest chromatic neighbour). Thus, a UV/green bicolour display of $9 \times 12$ pixels is obtained, covering $96^{\circ}$ in azimuth and $66^{\circ}$ in elevation, sufficient to cover most of the field of view of the median ocellus.

The driving circuitry is an extension of that described by van Kleef et al. (2005), using the concept described by Lindemann et al. (2003). A Pentiumbased lab computer is used to generate pseudorandom sequences that encode light intensity with 14-bit resolution. Encoded intensities were scaled by calibration factors that were determined for each individual pixel,

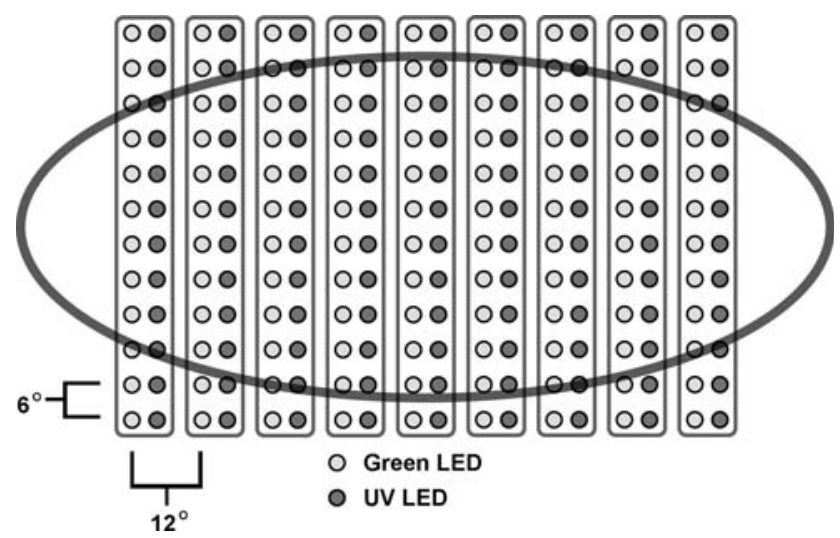

Fig. 1 Schematic diagram of the wide field LED display used for white noise analysis. The display consists of 9 columns, each column containing 12 rows of green and UV LED pairs. Resolution is higher $\left(6^{\circ}\right)$ in the vertical direction than the horizontal direction $\left(12^{\circ}\right)$ to match the receptive fields of photoreceptor neurons. The head of the dragonfly is oriented such that the overall field of view of the median ocellus (grey ellipse), which extends $120^{\circ}$ in azimuth and $60^{\circ}$ in elevation, is centred on the stimulus. Note that the figure is a schematic projection, with angular distances depicted as constant size. In reality, LEDs are arranged around the surface of a sphere of radius $120 \mathrm{~mm}$ to equalise light outputs of all pixels to within $5 \%$. Data were sent via a parallel port to a microcontroller (Isopod, NewMicros), which converted them into serial format, driving nine pairs of 14-bit D/A converters (Analog Devices AD5532HS), with each pair of D/A converters driving one column of LEDs. Individual pixels within each column are independently addressed by feeding the D/A converter outputs, via 12-channel, single pole multiplexers, into sample-and-hold voltageto-current converters that drive the LEDs. The refresh rate is $625 \mathrm{~Hz}$, allowing presentation of stimuli at a frequency higher than that which the ocelli can resolve (Ruck 1958). The usage of sample-and-hold circuitry allows all LEDs to be switched on continuously, with a maximum intensity of $\sim 10^{14}$ photons $\mathrm{cm}^{-2} \mathrm{~s}^{-1}$. This is sufficient to obtain an overall brightness that matches an equivalent patch of daylight sky.

We displayed a sequence of random contrast patterns at times $t_{n}=n \Delta t$, where $\Delta t=1.6 \mathrm{~ms}$ and $n=0, \ldots$, 12,511 where at each location the contrast values were uniformly and independently distributed in the range \pm 0.82 . The cell response $y(\mathrm{mV})$ to this sequence was recorded and the first-order space-time Wiener kernels $h(t, \theta, \phi)$, which describe the cells linear temporal response to contrast stimulus at each point of elevation $(\theta)$ and azimuth $(\phi)$, were obtained by fitting this response to the following equation using multiple linear regression (James et al. 2005; van Kleef et al. 2005).

$$
y\left(t_{n}\right)=f_{0}\left(t_{n}\right)+\sum_{\tau_{1}=0}^{\tau_{m}} \sum_{\theta=-33}^{33} \sum_{\phi=-48}^{48} h(\theta, \varphi, \tau) c\left(\theta, \varphi, t_{n}-\tau\right) .
$$

In Eq. $1 f_{0}$ includes a constant offset and terms for nuisance data modelled as a polynomial trend in time and $50 \mathrm{~Hz}$ hum (van Kleef et al. 2005). The contrast at each point of elevation and azimuth is defined as $c=\left(I-I_{0}\right) / I_{0}$, where $I$ is the intensity level at that point and $I_{0}$ is its mean temporal intensity. The optimal memory of this model $\left(\tau_{\mathrm{m}}\right)$ was obtained using the cross-validation methods outlined in van Kleef et al. (2005). The receptive field was taken to be the spatial profile that occurs at the time step when the maximal value of $h$ occurs.

\section{Staining and histology}

Upon successful completion of an intracellular recording, an attempt was made to identify the unit by iontophoretic injection of 5-10 nA negative current (positive current where the electrode was backfilled with Rhodamine Dextran) for a period of up to $60 \mathrm{~min}$. 
Following current injection, the brain and suboesophageal ganglion were dissected in fixative $(3.7 \%$ paraformaldehyde in phosphate buffered saline), transferred to $3.7 \%$ formaldehyde in methanol for $45 \mathrm{~min}$, dehydrated through an ethanol series and mounted in methyl salicylate. Filled neurons were observed under a Nikon Optiphot epifluorescence microscope. When the quality of the filled neuron was acceptable the neuron was optically sectioned on either a BioRad LaserSharp2000 or a Leica SP2 UV confocal microscope.

Two stained neurons from a single preparation were reconstructed by a combination of threshold segmentation and semi-automatic tracing of fine dendritic structures in the program Amira (Mercury Computer Systems). Additionally, the anterior parts of all other visible L-neurons in the brain were reconstructed as completely as possible by manually tracing the profiles of these cells using autofluorescent footprints present in the specimen. The outline of the brain from this preparation was also obtained by threshold segmentation. Surfaces were generated from the traced outlines, after which each axon profile could be clearly identified as a specific neuron type. Reconstructions for unstained neurons could be obtained only for the anterior region of the brain. Visualisation of more posterior brain regions required the use of an objective of lower numerical aperture, but this did not yield sufficient contrast to reliably trace autofluorescent footprints.

Stained neurons were reconstructed at the highest resolution possible which required imaging the neurons as separate anterior and posterior regions. The generated surfaces were then aligned with respect to each other and to landmarks present in lower magnification reconstructions of the neurons. Similarly, reconstructions of the brain and optic lobes were obtained by imaging several regions separately, generating a surface by threshold segmentation for each region and then manually aligning the surfaces relative to each other.

To greatly reduce surface polygon count and to join congruent brain and neuron surfaces together, all reconstructions were imported into Silo (http:// www.nevercenter.com), where the outlines of the brain and unstained neurons were manually redrawn. Images of reconstructions were obtained by exporting all surfaces to Blender (http://www.blender3d.com) and rendering with the YafRay renderer (http:// www.yafray.org).

\section{Results}

The lens, plexus area and the ocellar nerve of the dragonfly median ocellus are clearly divided by the midline of the brain into left and right halves. The left and right halves of the ocellar plexus are hereafter referred to as left and right lobes, as seen from a ventral view of the brain. Two species of closely related dragonflies were used in this study, namely, Hemicordulia tau and Hemicordulia australiae. H. australiae were used only for analysis of electrophysiological responses to point sources of light. In this respect no differences were observed between the two species.

Our results are based on over 87 recordings of units in the ocellar nerve, of which 48 were successfully identified by iontophoretic dye injection. Based on this material, we believe there to be a total of 11 L-neurons present in the dragonfly median ocellus. This set consists of five paired neurons that form mirror images of each other on either side of the brain, and one unpaired, bilaterally symmetrical neuron. The naming convention used is the original nomenclature provided by Patterson and Chappell (1980) and expanded upon by Mobbs et al. (1981). In this nomenclature neurons are referred to as contralateral if the axon projects from one lobe of the median ocellus to the contralateral half of the brain, and ipsilateral if the axon projects from one lobe of the median ocellus to the ipsilateral half of the brain.

Anatomical profiles of individual L-neurons

$M C_{1}$ and $M C_{2}$

There are two types of MC (median, contralateral) neuron in the dragonfly median ocellus, both of which have similar morphological features (Fig. 2a, e). Both types occur as symmetrical pairs, with one member of the pair innervating only the left lobe of the ocellar plexus and the other branching only to the right. All MC neurons possess extensive dendritic branching patterns and innervate almost an entire lobe of the ocellar plexus. The dendrites collate to form thick axonal trunks which then cross the midline of the brain shortly after exiting the ocellar nerve. The axons curve to run along the dorsal aspect of the brain and terminate in the posterior slope (PSL). Shortly after leaving the ocellar nerve and entering the brain proper, these neurons, and all other L-neurons, give rise to a slender neurite which runs in a loop towards the anteromedialmost aspect of the brain. The neurite connects with the cell body, which itself is located in close proximity to the pars intercerebralis.

While both $\mathrm{MC}_{1}$ and $\mathrm{MC}_{2}$ have similar general features, there are consistent differences in morphology between the two types of neuron. This is documented in Fig. 3a which shows the profiles of both 

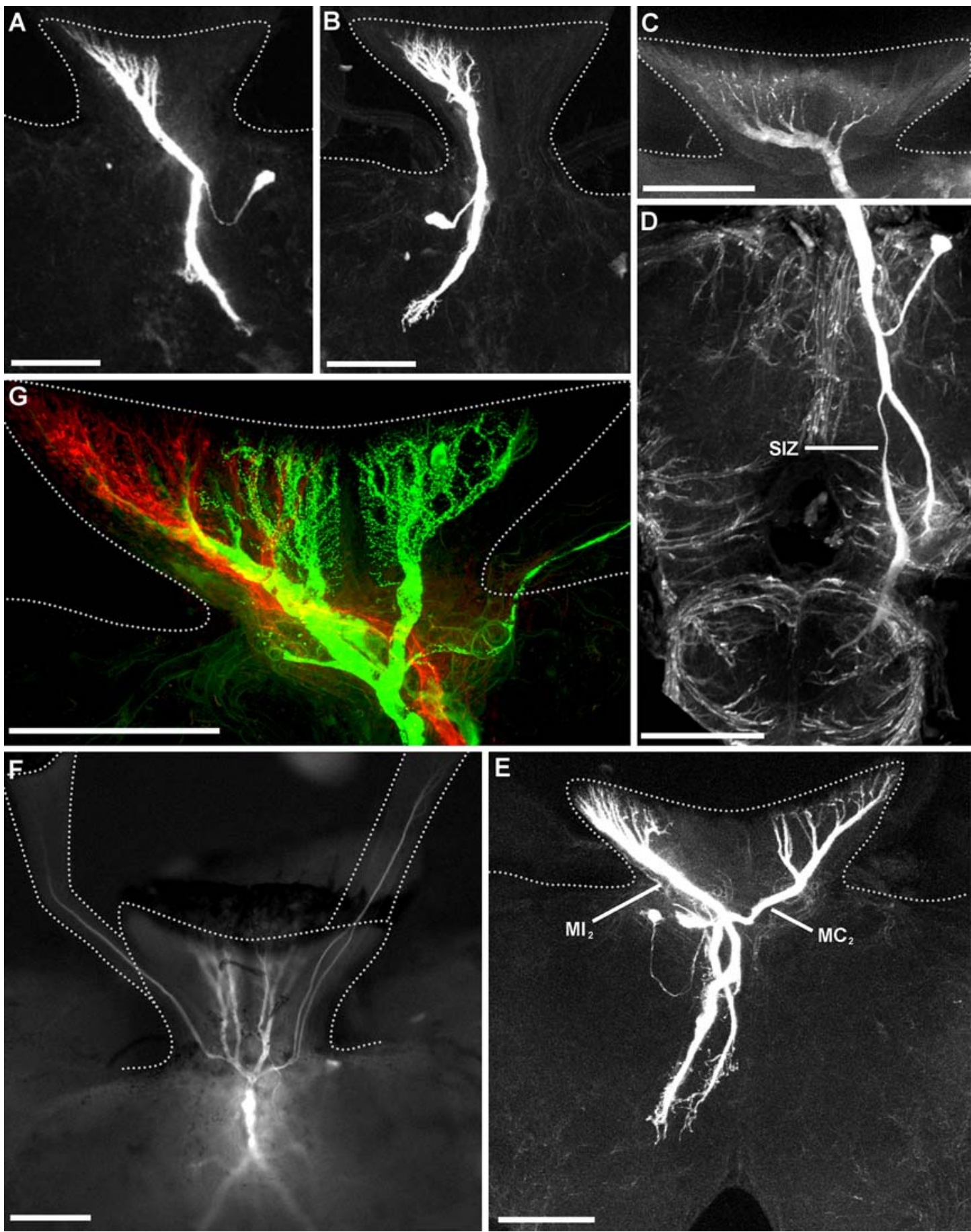

Fig. 2 Ventral views of stained median ocellar L-neurons in the dragonfly brain from six different preparations of Hemicordulia tau or H. australiae. All types of L-neuron present in the median ocellar nerve are represented and were identified anatomically by iontophoretic injection of dye after recording from the unit. a One of the paired $\mathrm{MC}_{1}$ neurons. b One of the paired $\mathrm{MI}_{1}$ neurons. c The dendritic branching pattern of one MD neuron. d The axonal branches of MD. The presumable site of spike initiation is shown (SIZ). e A double fill of two neuron types, $\mathrm{MC}_{2}$ and $\mathrm{MI}_{2}$. f The unpaired, bilaterally symmetrical $\mathrm{MB}$ neuron. g The dendritic branching of MB in the median ocellus at high resolution after staining with Lucifer Yellow (green). A second

cell $\mathrm{MC}_{1}$ has also been filled with Rhodamine Dextran (red) showing the two neurons branching to different regions within the ocellar neuropile. Note that a third cell $\left(\mathrm{MC}_{2}\right)$ has been partially filled with Lucifer Yellow which gives the impression of asymmetry across the midline of the brain in the green channel. With the exception of (f) all images are maximum intensity projections from a series of optical sections taken through the brain. $\mathbf{f}$ is an image taken under direct epifluorescence. Dotted grey lines depict anterior outlines of the brain and median ocellar nerve and plexus (not including retina). In $\mathbf{f}$ outlines of the lateral ocellar nerves have also been drawn. Scale bar all $200 \mu \mathrm{m}$ 
types of MC neuron which were recorded from different sides of the median ocellar nerve of the same preparation. The axon of $\mathrm{MC}_{1}$ is larger and within the ocellar plexus this neuron has a much stubbier appearance, typically forming a small number of thick dendritic branches which give off many short, fine dendritic branches over a small area. $\mathrm{MC}_{2}$ on the other hand has a much smoother transition from the small dendritic branches to the large diameter axonal trunk. These neurons are also clearly differentiated on examination of the dendritic branching pattern as seen from an anterior view of the brain (Fig. 3b). The dendrites of $\mathrm{MC}_{1}$ project relatively evenly over a large range of the ocellar plexus, with a general trend for branches to project ventrally into the plexus. In $\mathrm{MC}_{2}$
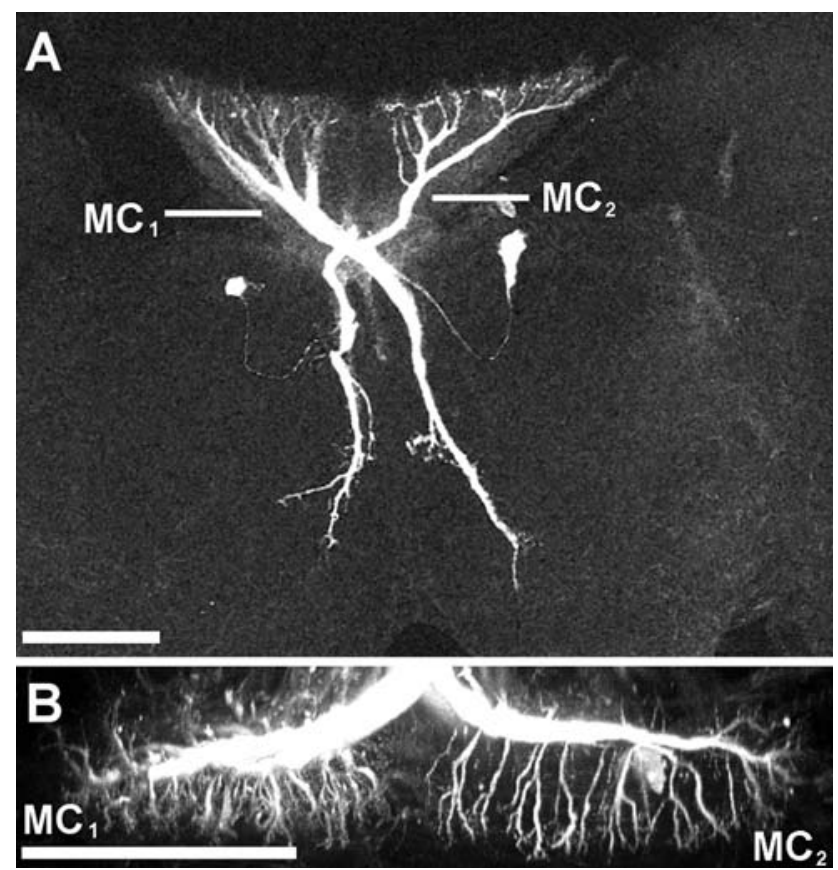

Fig. 3 The two types of MC neuron in the dragonfly median ocellus. Both neurons were stained independently in the same preparation. a Ventral view of the brain and the two neurons. $\mathrm{MC}_{1}$ is shown projecting from the left side of the ocellar plexus to the right side PSL, while $\mathrm{MC}_{2}$ is shown projecting from right to left, respectively. Differences in shape and termination areas are observable between the two types of neuron. $\mathrm{MC}_{1}$ is larger, stubbier and terminates in a more posterolateral area of the PSL than $\mathrm{MC}_{2}$. $\mathbf{b}$ The same pair of neurons from an anterior view of the median ocellar plexus. $\mathrm{MC}_{1}$ is again on the left hand side and shows a relatively evenly dispersed branching pattern around the main trunk of the neuron. $\mathrm{MC}_{2}$ sends off a series of branches to the ventral extremes of the neuropile (bottom) while only a few short branches project dorsally (top), resulting in a pronounced asymmetry around the dendritic trunk. Both images are maximum intensity projections from a series of optical sections through the brain. b has been virtually resliced in the frontal plane from a series of ventral images before projection. Scale bars $200 \mu \mathrm{m}$ this effect is much more pronounced and long dendritic branches may be seen leaving the main trunk of the neuron to run ventrally at an almost perpendicular angle before terminating near the ventral extreme of the ocellar plexus. The axon of $\mathrm{MC}_{2}$ also terminates in a more anteromedial region of the PSL than does $\mathrm{MC}_{1}$.

\section{$M I_{1}$ and $M I_{2}$}

As with the MC neurons, both types of MI (median, ipsilateral) neuron also occur as symmetrical pairs with one member of the pair innervating the left lobe of the ocellus plexus and the other the right (Fig. 2b, e). Shortly after leaving the ocellar neuropile the MI neurons form single, thick dendritic trunks, which curve first medially to run towards the midline of the brain before turning to run back posterolaterally. This can result in an interwoven appearance when viewed in association with the MC neurons as in Fig. 2e.

As with the $\mathrm{MC}$ neurons, $\mathrm{MI}_{1}$ branches extensively within ocellar plexus, covering the majority of one lobe (Fig. 2b). In contrast, the $\mathrm{MI}_{2}$ neurons are unique in that they branch only to the lateral extremes of the ocellar plexus (Fig. 2e). The axons of both MI type neurons run in close association with each other, with $\mathrm{MI}_{2}$ running in a slightly more lateral position and terminating more laterally in the PSL than $\mathrm{MI}_{1}$.

$M D$

Two MD neurons also form a symmetrical pair across the midline of brain. These two neurons are the largest, most prominent neurons within the median ocellus. Surprisingly, given their size and prominent position within the ocellar nerve, this neuron type has not been previously described. However, from cobalt staining initiated from the ligated ocellar nerve, Patterson and Chappell (1980) did describe the existence of nerve fibres within the brain with morphology similar to the posterior axonal branches of MD as described below. Patterson and Chappell (1980) tentatively assigned these axonal branches to two separate third-order ocellar neurons (OI1 and OI2), which they believed to originate from the PSL and were stained by transsynaptic migration of cobalt ions from an ocellar Lneuron. In light of the present results it appears that these two neurons are, in fact, axonal branches of one and the same neuron, which is itself an L-neuron rather than a third-order neuron. We refer to this neuron type as MD (median, descending). A correlate of MD has been found in at least one other dragonfly species, namely Aeschna canadensis (R. Olberg, unpublished), indicating that it is not specific to Hemicordulia. 
The MD neurons are unique both in terms of morphology and physiology. Firstly, MD neurons are bilateral in that they innervate both lobes of the ocellar plexus, but in an asymmetrical fashion such that, for example, $\mathrm{MD}_{\mathrm{L}}$ (the $\mathrm{MD}$ neuron with axon and cell body on the left side of the brain) will innervate the entire right lobe of the ocellar plexus, but only the medial half of the left lobe of the ocellar plexus (Fig. 2c, d). Secondly, these neurons do not run along the dorsal-most aspect of the brain, but instead lie ventral to the other L-neurons and descend towards the posterior of the brain more rapidly. Lastly, unlike all other median ocellar L-neurons in the dragonfly these neurons do not contribute to the PSL. Midway through the brain each MD neuron bifurcates into two axonal branches. A thicker branch proceeds laterally to terminate in a confined area of the posterior deutocerebrum, while a second branch, running medially to the first, forms a narrow constriction (believed to be the site of action potential generation, see below) before expanding again prior to entering the suboesophageal connectives and continuing on to the suboesophageal ganglion or SOG (Fig. 2d). This neuron has been traced as far as the SOG, but due to limitations in dye diffusion it is currently unknown whether the axon terminates here or continues into the ventral nerve cord. Patterson and Chappell (1980) describe OI1 (considered here to be commensurate with the descending branch of MD) as descending into the ventral nerve cord, but were similarly unable to determine the end point of termination.

\section{$M B$}

The last of the 11 L-neurons in the dragonfly median ocellus is the single, unpaired neuron MB (median, bilateral). Of all dragonfly L-neurons this neuron has the most distinctive branching pattern. It was also the least frequently recorded neuron, with successful dye fills being obtained only twice. As previously described (Patterson and Chappell 1980; Mobbs et al. 1981), MB descends down the midline of the brain with bilaterally symmetrical branches projecting to both lobes of the ocellar plexus. Within the brain this neuron gives off two small collateral branches which project to each of the two lateral ocelli (Fig. 2f). The cell body is believed to lie on the midline of the brain.

Under high magnification it can be observed that MB innervates only the medial aspects of each ocellar plexus lobe (Fig. 2g). Partial innervation of the ocellar plexus has also been observed in the lateral ocelli, although it could not be determined which region of neuropile is innervated by this neuron. From Fig. $2 \mathrm{~g}$ it is also clear that numerous, small, fine, branches extend towards the midline of the median ocellar plexus and nerve. Within the brain, just prior to bifurcation to the PSLs, two slender collaterals can also be seen extending from the axonal trunk and running laterally a short way into the brain before terminating near the dorsal surface.

\section{Three-dimensional reconstructions}

Reconstructions of the L-neurons were obtained to further analyse their geometry and spatial relationships within the brain and median ocellus. All reconstructions were obtained from the same preparation. This preparation had first been used for intracellular recording, and two neurons $\left(\mathrm{MC}_{2}\right.$ and $\left.\mathrm{MI}_{2}\right)$ recorded from opposite sides of the median ocellar nerve were stained independently by iontophoretic injection of Lucifer Yellow. Full reconstructions of these two neurons were obtained. Partial reconstructions of the remaining neurons were obtained by manually tracing autofluorescent footprints present in the specimen.

After intracelluar recording, dissection and tissue processing, the brain of this preparation was optically sectioned at various magnifications, locations and laser intensities by confocal microscopy. L-neurons (like other invertebrate interneurons) produce less autofluorescence upon excitation by light of blue or green wavelengths than the surrounding brain tissue and axon profiles therefore appear dark in optical sections of the median ocellar nerve (Fig. 4a). Usually the relatively small contrast difference between an unstained neuron and the surrounding tissue allows only limited resolution of neuron fibres. However, due to the very large axonal diameters of the L-neurons, optical sectioning of the median ocellar nerve at high laser power and gain allows tracing of axon profiles in three dimensions.

Reconstructions of all L-neuron types present in the median ocellar nerve are shown in Fig. $4 b-d$. The median ocellar retina is surrounded by an opaque pigment layer which prevented accurate delineation of its shape and could therefore not be reconstructed. It is unclear to what extent the pigment layer also sheathes or obscures the anterior of the ocellar plexus; however, the length of the median ocellar nerve appears artificially short in reconstructions from fluorescence microscopy because of this sheathing layer. Reconstructions of unstained neurons are less complete than those of the two stained neurons as the contrast present in unstained axonal profiles is not sufficient to allow resolution of fine dendritic details. Nevertheless, tracing autofluorescent footprints allowed three- 

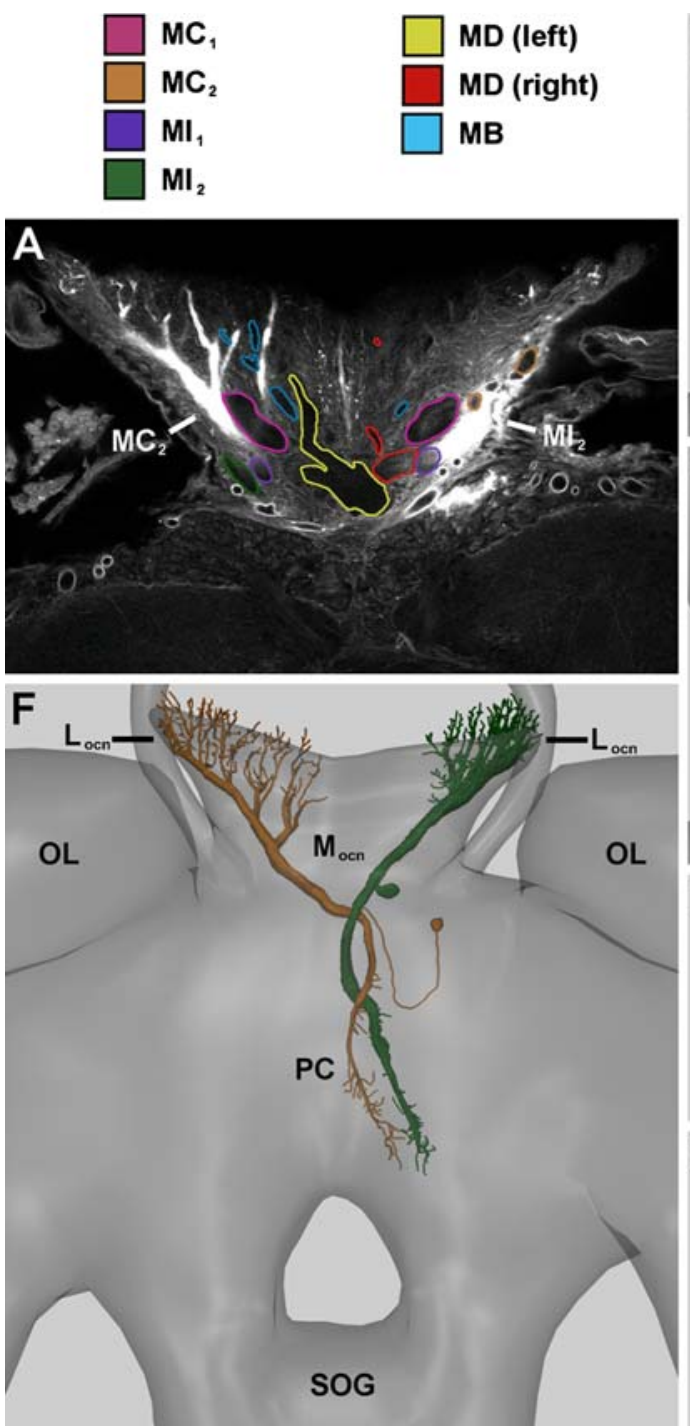

Fig. 4 Three-dimensional reconstructions of L-neurons in the dragonfly median ocellus. Reconstructions were performed on a single preparation of Hemicordulia tau from which two neurons were stained by iontophoretic injection of Lucifer Yellow (the same preparation as in Fig. 2e). a Ventral view of an optical section through the median ocellar nerve. The two stained neurons, $\mathrm{MC}_{1}$ on the left and $\mathrm{MI}_{2}$ on the right, are clearly visible as strongly fluorescent structures. Autofluorescent footprints of unstained L-neurons are shown as coloured contours. To avoid ambiguity the left and right members of the bilateral MD neurons are shown as yellow and red respectively. Other paired neurons innervate one lobe of the median ocellus only and therefore both members of the pair are shown in the same colour. Reconstructions of the stained and unstained neurons within the median ocellus are shown from a ventral viewpoint in

dimensional description of the axons of all large axon profiles in the median ocellus of a single preparation, which allowed verification of the number of L-neurons present in the dragonfly median ocellus. Cell stainings from multiple preparations suggested the presence of at least six L-neuron types in the dragonfly median
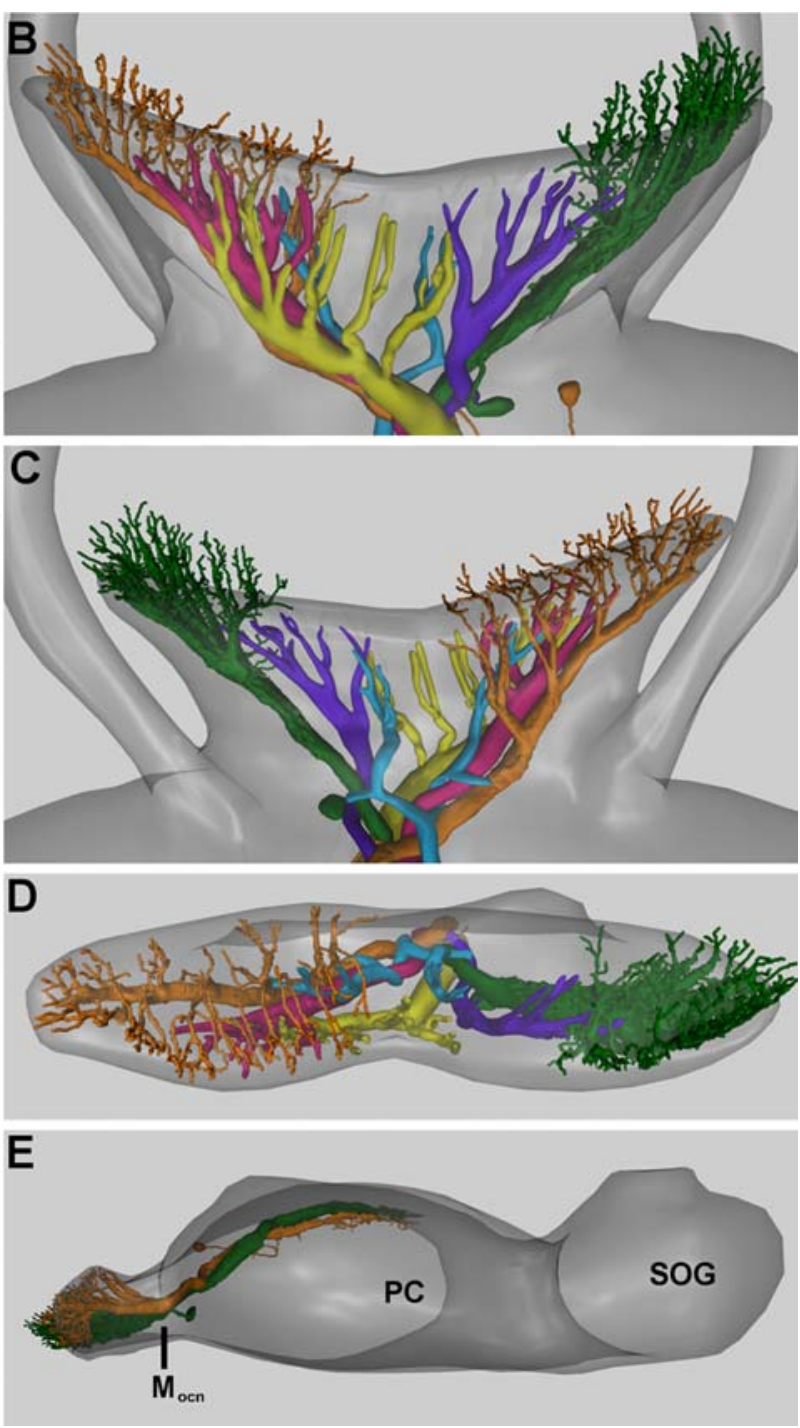

(b), a dorsal viewpoint in (c) and a frontal viewpoint in (d). For clarity only the left member of each pair of neurons is shown (where left refers to the location of the axon or cell body of the neuron within the brain from a dorsal view). For simplification the lateral ocellar nerves and brain are not shown in (d). The fully reconstructed stained neurons are shown from an orthographic lateral view in (e), (lateral ocellar nerves and optic lobes not shown) and from a ventral viewpoint in (f). In (d) and (e) ventral is bottom and dorsal is top. Note that the apparent projection of neurons anteriorly from the median ocellar nerve into the retina (not shown) may be an artifact due to the presence of an opaque pigment layer sheathing this region. $M_{\mathrm{ocn}}$ median ocellar nerve; $L_{\text {ocn }}$ lateral ocellar nerve; $P C$ protocerebrum; $O L$ optic lobe; $S O G$ suboesophageal ganglion

ocellus and all six types have been traced in this preparation. Additionally, no other large axon profile was observed to innervate the ocellus suggesting that additional L-neuron types are not present. A number of smaller axonal profiles could be observed within the median ocellar nerve, however, these were of much 
smaller diameter than the L-neurons and may be either S-neurons or smaller dendritic branches of an L-neuron that could not be associated to an axonal trunk. Dark profiles with brightly fluorescent borders are trachea rather than neuron profiles.

Reconstruction of the two stained neurons $\mathrm{MC}_{2}$ and $\mathrm{MI}_{2}$ shows that although the two neurons clearly project to different regions of the ocellar plexus in the horizontal plane (Fig. 4c), both neurons innervate a wide area of the ocellar plexus in the vertical plane (Fig. 4d). It can also be seen that both neurons run along the dorsal-most aspect of the brain and terminate at a similar location in the vertical (Fig. 4e) but not the horizontal axis (Fig. 4f).

Physiological responses to pulses of light

from a point source

\section{Time course}

General For the purpose of comparison with previous research (the majority of which precedes 1982) the responses of dragonfly L-neurons to impulses of light are described. An example of a typical response to a light pulse is shown in Fig. 5a. Approximately $10 \mathrm{~ms}$ after stimulus onset a rapid hyperpolarizing transient is observed, the amplitude of which often exceeds $30 \mathrm{mV}$. After the initial transient the membrane potential returns relatively slowly to a less hyperpolarized plateau, which is maintained for the length of the stimulus. On stimulus offset, an initial fast off transient (FOT) is usually observed with a latency of approximately $10 \mathrm{~ms}$. A small number of oscillations around the resting membrane potential of the recorded unit may follow the FOT before a prolonged depolarizing afterpotential (PDA) is observed. The PDA typically begins approximately $100 \mathrm{~ms}$ or more after stimulus offset and may last for upwards of $500 \mathrm{~ms}$.

Although the step responses of all cells consisted of similar components, the amplitude of each component could vary considerably between recordings. In some recordings, for example, the hyperpolarized plateau may appear almost as large as the hyperpolarizing transient, while in others the entire 'on' response appeared greatly reduced in comparison to the 'off' response. This did not appear to relate to neuron type (with the exception of MD, see below) or the quality of the recording. Recorded units were required to show large $(>20 \mathrm{mV})$, stable responses to light impulses for approximately 2 min with little fluctuation in resting membrane potential before intracellular responses were recorded. Obtaining stable recordings was seldom problematic, presumably due to the large size of the L- neurons. Intracellular units were often held for periods of greater than $2 \mathrm{~h}$ with little observable fluctuation in responses over the recording period.

Comparison of the response from a green and UV point source of light positioned at a location that was deemed to produce maximal response almost invariably resulted in differences in the latency (typically 5-10 ms slower with green light) and waveform of the response. However, it is unclear as to whether these differences are attributable to genuine differences in chromatic mechanisms or whether they are attributable to sensitivity differences to green and UV light, given that the necessary controls for the effects of light adaptation are very difficult to achieve.

The role of compound eye input on the step response was assessed by occluding the compound eyes, either by painting with opaque black paint, or covering with a mask made of aluminium foil or brass. In some cases the mask could be removed while recording from a unit such that the effect of compound eye occlusion could be directly compared by analysis of step responses before and after mask removal. In no case did
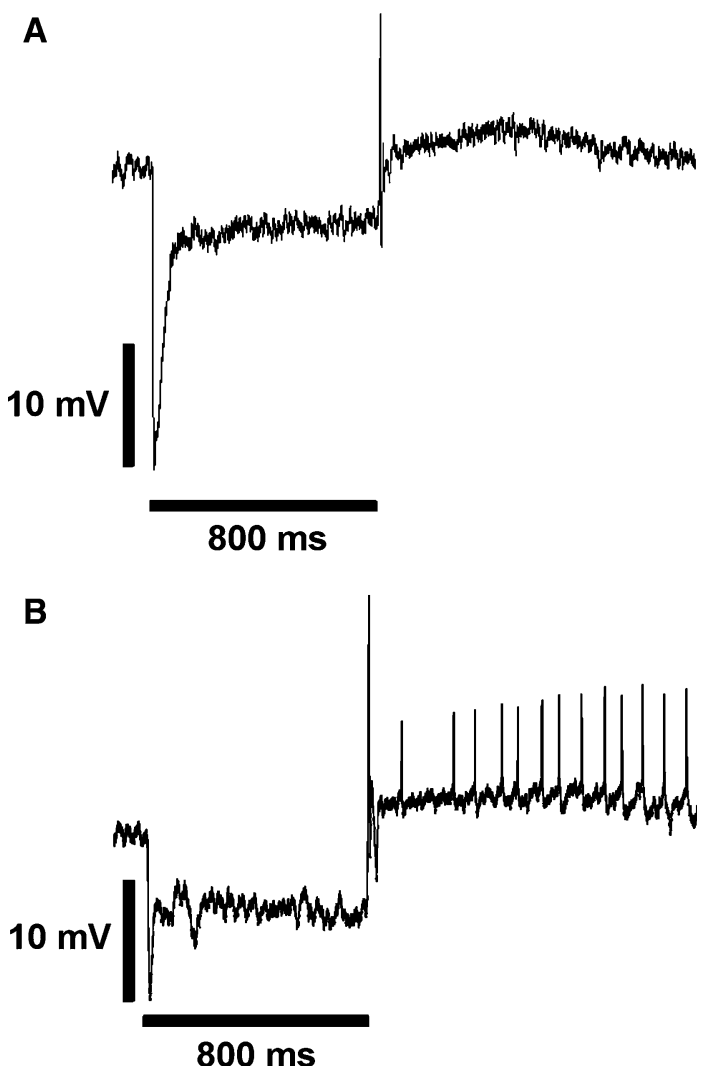

Fig. 5 Characteristic responses of L-neurons. a Representative response of an L-neuron $\left(\mathrm{MC}_{2}\right)$ to a square wave UV light impulse of $800 \mathrm{~ms}$ duration. b shows the usual response of MD to the same stimulus 
occlusion of the compound eyes visibly affect the step response of the unit.

Specific characteristics of MD The paired MD neurons are physiologically distinct from the other L-neurons of the dragonfly median ocellus and will therefore be considered separately. In general, the responses of this neuron to a brief impulse of light were very similar to those described above for other L-neurons. However, unlike all other L-neurons, these neurons produce regenerative action potentials. In no case was any other L-neuron type found to spontaneously produce regenerative potentials, nor could such action potentials be induced by stimulation with light, or the injection of current. The firing frequency of MD neurons was found to differ markedly between preparations. In some recordings of MD the neuron spontaneously produced trains of action potentials at rates of up to 60 spikes per second independently of visual stimulation. In other recordings, however, action potentials were only elicited in a brief period following a reduction in light intensity. As shown in Fig. 5b, in preparations with low or zero spontaneous firing frequency, action potentials were only observed riding the slow depolarization following light offset. In preparations with higher spontaneous firing frequency light onset reduced, while light offset increased the spike frequency. The firing frequency of MD could be similarly affected by the injection of current into the unit such that the injection of positive current increased firing frequency, while the injection of negative current reduced it. Post-inhibitory rebounds are also observed following periods of negative current injection or prolonged exposure to bright light. Action potentials are presumed to originate posterior to the narrow constriction of the descending axonal branch of MD (Fig. 2d), due to the low-pass filtering nature of small diameter axons.

MD was also found to respond to modalities other than vision. Mounting the dragonfly in a position where the wings were left free allowed the effect of wing movement (self-induced or forced) on firing frequency to be assessed. In some cases no effect of wing manipulation occurred. In other cases, however, manually depressing the wings downwards appeared to produce one or more action potentials or increase firing frequency, while elevating the wings appeared to result in phasic hyperpolarizations or decrease firing frequency. Similarly, the presence of wind typically resulted in a reduction of spontaneous firing frequency as established by gently blowing a stream of air funnelled through a pipette around the head and neck of the dragonfly.
Dependence of responses on angular position of point source

By mounting a pair of green and UV LEDs on a rotatable cardan arm, square wave light pulses of variable duration and repetition rate could be delivered from any direction within the median ocellar field of view. Responses to light pulses of constant intensity typically resulted in saturation so that no clear correlation between stimulus direction and the dendritic branching pattern of a neuron could be established. However, by measuring intensity-response functions it was possible to use a constant response criterion, similar to that used by Wilson (1978) in ocellar L-neurons of locusts. Under those conditions, the presence of directional sensitivity differences could be established, but only for large changes in azimuth angle, while sensitivity gradients in elevation could not be established. This is inconsistent with the presence of spatial resolution in the median ocellar receptor neurons of $H$. tau (van Kleef et al. 2005), suggesting that the combination of adaptation in both the receptor neurons and second-order neurons is sufficiently fast to mask changes in sensitivity associated with changes in angular position. Light flashes from point sources against a dark background are rather extreme stimuli for a system that is capable of responding over a very wide range of intensities (Stange 1981).

\section{Receptive Fields by Wiener kernel analysis}

It follows from the above that it is necessary to determine angular sensitivities by a stimulus regime that is more akin to natural conditions, namely a multielement, two-dimensional array of light sources, in which the preparation can be kept at a defined level of light adaptation that simulates natural conditions and in which individual pixels can be modulated by small contrasts. White noise analysis of simultaneously modulated pixel intensities fulfills these criterion. In the present investigation there were two major reasons why white noise analysis was particularly advantageous for analysing receptive fields of the L-neurons. First, it avoids the confounding effects of adaptation. Second, it produces the small-signal or linear response, thereby minimising non-linear interactions between cells.

Figure 6 provides an illustrative example of the generation of receptive field maps by white noise analysis. The intracellular response to random intensity modulation simultaneously across all pixels in the display, along with the contrast modulation of a single UV LED is shown in Fig. 6a. For each pixel in the display, a linear kernel is then obtained using Wiener kernel 
analysis (Fig. 6b). Wiener kernel methods have been used extensively to quantitatively model the insect visual system (Marmarelis and McCann 1973; Sakai et al. 1988). Although reverse correlation methods have traditionally been used to calculate Wiener kernels (Lee and Schetzen 1965), more recently multiple linear regression has been employed for this task (James et al. 2005; van Kleef et al. 2005). Combined analysis of linear kernels at all spatial locations simultaneously at a single point in time results yields a receptive field map (Fig. 6c). Receptive fields are always calculated from the point in time at which the amplitude of response is maximal.

Receptive field contour maps and acceptance angle outlines for representative examples of all L-neuron types are shown in Fig. 7. The receptive fields shown are calculated from responses to pseudorandom modulation of UV light in the presence of concurrent pseudorandomly modulated green light. Kernel analysis of responses to green light sequences in the presence of concurrently modulated UV light never generated receptive field maps distinguishable from noise. Comparison of the contour outlines shown in Fig. 7, with morphological analysis of the retinal projections of the L-neurons shown in Fig. 2, provides an excellent match, in that the extent of the receptive fields obtained corresponded closely to the extent of dendritic branching within the ocellar neuropile. Some variation between receptive field maps of the same neuron type occurs between preparations (see Table 1), but this variation was found to be small. For those neurons that cover almost an entire half of the ocellar plexus, such as $\mathrm{MC}_{1}$, $\mathrm{MC}_{2}$ and $\mathrm{MI}_{1}$, the $50 \%$ maximum response contours are constrained to one half of the median ocellar field of view. The lateral neuron $\mathrm{MI}_{2}$ samples only extreme lateral visual space, MD samples the full extent of one side and the median part of the other, whereas MB covers only the medial region of visual space.

Table 1 shows the mean location of centre points and acceptance angles averaged over all recordings of identified L-neurons. From this it can be seen that in elevation, the mean positions of the receptive fields are consistently a few degrees (between $2.7^{\circ}$ and $6.8^{\circ}$ ) above the equator, and there is no evidence that different cell types sample different elevations. The acceptance angles in elevation are approximately $20^{\circ}$, which is not much more than the mean acceptance angle of $15^{\circ}$ observed in recordings from dragonfly median ocellar photoreceptors (van Kleef et al. 2005). The elevation range covered by $\mathrm{MD}$ in particular appears to be especially narrow in comparison to the other L-neurons, such that this neuron samples a
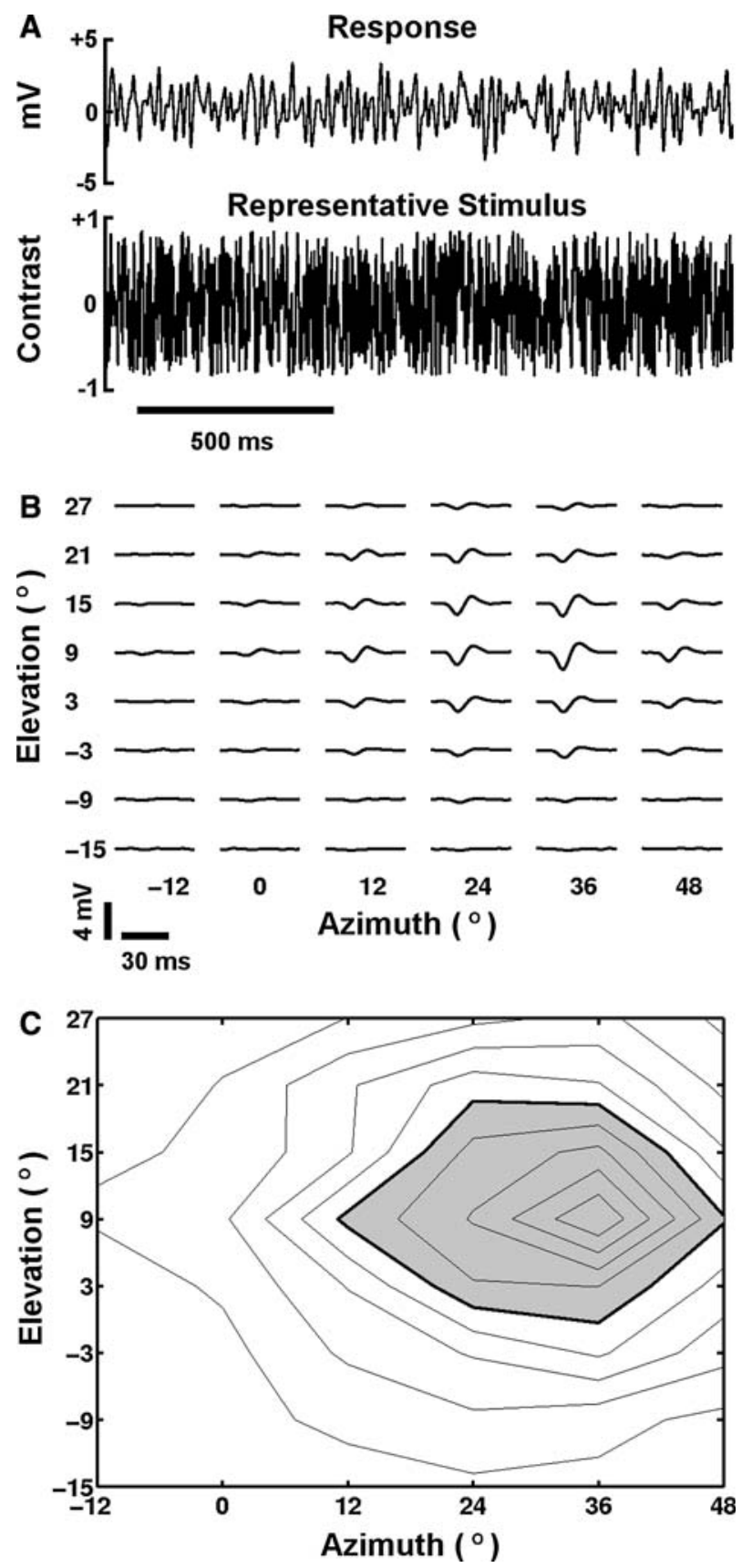

Fig. 6 The procedure for measuring the receptive fields of second-order neurons. a The recorded response to a random wide field stimulus and the corresponding UV contrast stimulus from a single pixel in the wide field LED array. b First-order temporal kernels for pixels in the range of $-12^{\circ}$ to $+48^{\circ}$ in azimuth and $-15^{\circ}$ to $+27^{\circ}$ in elevation. These were generated from a white noise analysis of the contrast stimuli at each pixel and the recorded cell response in (a). Note that kernels have been scaled to represent the intracellular response to a $1 \mathrm{~ms}$ impulse of unit sized contrast. c The receptive field generated from the kernels shown in (b). Maximum response occurs at azimuth $=36^{\circ}$, elevation $=9^{\circ}$ and $t=16 \mathrm{~ms}$. Contours show $10 \%$ increments of maximum response from all kernels at $t=16 \mathrm{~ms}$. Responses at or above $50 \%$ of the maximum response (acceptance angle) are shown shaded in grey 

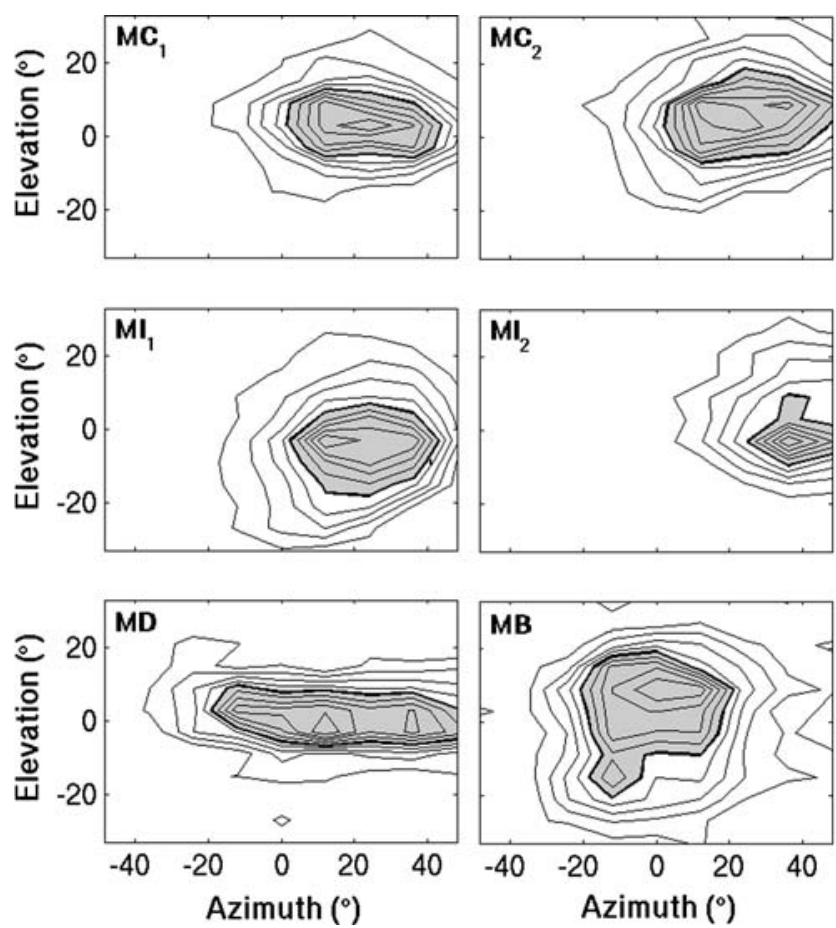

Fig. 7 Representative examples of two-dimensional receptive field maps of each L-neuron present in the dragonfly median ocellus. Linear spatiotemporal impulse functions were estimated by Wiener kernel analysis of intracellularly recorded responses to pseudorandom UV and green light sequences. Receptive fields were obtained from spatiotemporal impulse response functions at a delay deemed to produce maximal response (between 8 and $12 \mathrm{~ms}$ ). The median ocellus is centered on the wide field stimulus such that the optical axis of the eye lies at the point $(0,0)$ with positive going elevation values representing the upwards direction. For comparison all receptive fields are shown for the neuron of each pair sampling the right side of visual space (with the exception of the unpaired bilateral neuron $\mathrm{MB}$ ). Contour maps of the full receptive field are shown in black outlines with responses at or above $50 \%$ of the maximum response shown shaded in grey narrow streak of visual space slightly above the equator of the eye.

Table 1 also provides a measure of the reproducibility of receptive field sizes and locations. In general, variation in the obtained values is small with neurons of the same type consistently showing similar receptive fields. A degree of error may have been introduced by inaccuracies in the consistent alignment of the median ocellus with the centre of the stimulus, by anatomical distortion produced by stabilisation of the ocellar nerve with a supporting spoon, or by the introduction of an electrode into the nerve. The extent to which this variation is attributable to experimental procedure and not to morphological variation inherent in the ocelli is impossible to determine.

\section{Discussion}

\section{Anatomy}

\section{Dragonfly L-neurons}

The anatomy of the L-neurons in the dragonfly median ocellus has been the subject of two previous investigations. From cobalt fills of the ligated median ocellar nerve, Patterson and Chappell (1980) provided a detailed description of the axonal profiles of five types (nine total) of L-neurons in the median ocellus of Anax junius and Aeschna tuberculifera. At that time, the dendritic arborizations of only one of these neuron types could be described. Mobbs et al. (1981) later provided descriptions of four further neuron types from iontophoretic injection of 16 ocellar units in Aeschna tuberculifera, Sympetrum rubicundulum and

Table 1 Mean receptive field centre points and acceptance angles of dragonfly ocellar L-neurons

\begin{tabular}{|c|c|c|c|c|c|}
\hline \multirow[t]{2}{*}{ Cell type } & \multirow[t]{2}{*}{$n$} & \multicolumn{2}{|c|}{ Centre point $\left(^{\circ}\right)$} & \multicolumn{2}{|c|}{ Acceptance angle $\left(^{\circ}\right)$} \\
\hline & & Azimuth & Elevation & Azimuth & Elevation \\
\hline $\mathrm{MC}_{1}$ & 8 & $23.8 \pm 4.6$ & $2.7 \pm 3.9$ & $40.5 \pm 9.9$ & $18.2 \pm 4.8$ \\
\hline $\mathrm{MC}_{2}$ & 4 & $20.3 \pm 14.4$ & $6.8 \pm 3.9$ & $45.5 \pm 20.7$ & $21.9 \pm 2.2$ \\
\hline $\mathrm{MI}_{1}$ & 5 & $28.8 \pm 4.1$ & $4.4 \pm 6.1$ & $34.2 \pm 7.3$ & $18.6 \pm 4.3$ \\
\hline $\mathrm{MI}_{2}$ & 3 & $41.2 \pm 2.4$ & $5.1 \pm 6.6$ & $13.2 \pm 5.5$ & $15.9 \pm 6.0$ \\
\hline MD & 3 & $11.4 \pm 2.8$ & $4.2 \pm 4.9$ & $68.0 \pm 5.2$ & $15.0 \pm 0.3$ \\
\hline MB & 1 & -0.3 & 4.2 & 39.7 & 31.9 \\
\hline
\end{tabular}

Values were taken only from preparations in which the recorded unit could be clearly identified by anatomy. The number of recordings of each cell type is shown as $n$. For each recording the centre of the receptive field and the acceptance angles were determined at the moment in time, in the spatiotemporal impulse response, at which the response amplitude was maximal (between 8 and $12 \mathrm{~ms}$ ). Centre points and acceptance angles are shown as mean \pm standard deviation (standard deviations could not be shown for MB as this neuron was recorded from only once with this stimulus type). Both centre point values and acceptance angles are likely to be underestimated for $\mathrm{MI}_{2}$ as the receptive field of this neuron consistently exceeded the azimuthal range of the stimulus. Values used to determine centre point values are absolute values such that the results given indicate the centre of receptive fields for neurons in the same half of the ocellar neuropile (increasing azimuth may be defined as left or right arbitrarily as the two halves of the ocellus are symmetrical). The point $(0,0)$ lies at the optical axis of the eye with positive going elevation values representing the upward direction 
Pachydiplax longipennis. These authors made no distinction between either the two contralateral neuron types or the two ipsilateral neuron types. The present results are based on successful dye fills from 48 ocellar L-neurons and provide the most complete description of the anatomical and physiological mappings of the Lneurons in the dragonfly median ocellus to date. An additional neuron type (MD) has been described, and details of the dendritic arborizations of all neuron types have been given. This has allowed distinction between similar neuron types, for example, $\mathrm{MC}_{1}$ and $\mathrm{MC}_{2}$, on the basis of anatomy. Additionally, the recognition that dragonfly L-neurons project to different areas of the ocellar plexus has consequences for the receptive fields of these neurons as described below.

From comparison of previous descriptions with our own findings in Hemicordulia tau and the closely related Hemicordulia australiae, it would appear that in general the number and morphology of L-neurons in the ocellus is well conserved over a wide range of dragonfly species. We believe that the dragonfly median ocellus contains six types (11 total) of L-neurons. On no occasion was a neuron of ambiguous morphology found, suggesting that additional L-neuron types are not present. This is further supported by manual tracing of L-neuron profiles obtained by confocal microscopy, which left no large axon profile unaccounted for.

With the exception of MD, all L-neurons terminate in the PSL neuropile. Studies on other insects have shown that the L-neurons terminate in close contact with large descending neurons which originate in the PSL and project down the ventral nerve cord, presumably outputting to motor ganglia (Strausfeld 1976; Guy et al. 1979). In the dragonfly, Patterson and Chappell (1980) describe the outputs of the L-neurons as being confined to one or two areas within the PSL in a manner that appears to be related to the location of the dendritic arborizations within the ocellus. Given that different L-neurons map different regions of visual space this suggests that a degree of spatial mapping is present in the outputs formed by the L-neurons within the PSL. Whether this information is utilised by the higher-order, presumably descending neurons of the PSL is open to investigation.

\section{Comparisons with other species}

\section{General}

Broad similarities in the neuronal organisation of the ocelli also exist between dragonflies and insects of different orders. Correlates of the bilateral neuron MB for example may be seen in the locust, cricket and bee (reviewed in Goodman 1981). However, in these insects the innervation pattern of the bilateral neuron is constrained to the median ocellar neuropile, although tri-ocellar neurons have been observed in the housefly (Strausfeld 1976) and two species of caddisflies (Hagberg and Nässel 1986). In contrast, neuron pairs directly connecting the median ocellus to one of the lateral ocelli have been found in several insects whereas this neuron type is absent in dragonflies. It is therefore possible that MB subserves a similar function to pairs of bi-ocellar neurones found in other species.

Interestingly, high resolution confocal microscopy of the dendritic arborizations of MB in the median ocellus reveals numerous fine dendritic endings projecting perpendicularly towards the midline of the ocellar plexus and running almost the entire length of the ocellar nerve. Possible synaptic targets in this region of the nerve are unknown as the ocellar photoreceptors do not appear to penetrate deep enough into the nerve to output onto these collaterals (Patterson and Chappell 1980), and no L-neuron appears to form dendritic arborizations in this area. The likely solution is that these branches form efferent output to, or receive afferent input from, one or more of the S-neurons present in the dragonfly ocelli. Currently no S-neurons have been anatomically identified within the dragonfly ocelli, meaning that this hypothesis cannot be tested at this time. It is known, however, that the dragonfly median ocellus forms a complex network of interactions where presynaptic terminals make contact with two postsynaptic units, and that this may include synapses between second-order neurons (Dowling and Chappell 1972). Simmons (1982b) has directly tested interactions between second-order neurons by dual electrode impalement and found evidence for interactions between probable S- and L-neurons. This lends itself to the suggestion that MB may function as a global illumination detector, which receives information about light levels from a wide variety of directions, and uses this to modulate the function of other ocellar or brain neurons, for example, for adjusting states of light adaptation.

\section{Variability}

The number of neurons stained in the present study permits some comments on the amount of variability present in the morphology of the L-neurons. Neurons of the same type, but stained in different preparations, were found to show variations in the fine branching pattern of both the dendrites and the terminal arborizations as well as variation in the location of the cell body and the course of the axon through the brain. 
However, this variation was typically small. Indeed, we observe that the ocellar L-neurons exhibit markedly less variability than has been found for other insects. Analysis of intrinsic variability of L-neuron morphology has been performed comprehensively in the locust (Goodman 1974), and to a lesser extent in the bee (Milde 1984). In both cases neurons often showed a high degree of 'normal' variation which includes differences in the fine branching patterns of the axons, while a minority of neurons showed major variations, which included axon projection into abnormal brain areas and the presence of additional branches projecting to ocelli where they were normally not present. In contrast, on no occasion did we observe such abnormalities in the axonal profile of a neuron in the dragonfly median ocellus. This leads us to suggest that ocellar morphology is more consistently preserved in the dragonfly than in other pterygote insects. It must be noted, however, that Goodman (1974) stresses the importance of large sample sizes when analysing variability as the prevalence of abnormalities is low. It also must be noted that insects from long-term cultures, e.g. locusts, may have increased variability due to inbreeding, whereas this is not problematic for dragonflies. A more comprehensive analysis of variability within the dragonfly ocelli would require large sample sizes combined with mass staining techniques to visualise multiple neurons in a single preparation.

\section{Physiology}

\section{Responses to light pulses}

General characteristics Physiological responses to light pulses were similar to those reported previously for the dragonfly ocelli (Chappell and Dowling 1972; Patterson and Chappell 1980; Mobbs et al. 1981; Simmons 1982b). There is considerable variation between recordings in the amplitude of the constituent components of the response, and this has also been described by other investigators (Klingman and Chappell 1978; Milde 1981). Waveform variation does not appear to be attributable to differences in stimulus wavelength (Mobbs et al. 1981), but may be a function of recording quality, damage caused by dissection or electrode insertion, or the location of the electrode tip. Conversely, the variation in observed responses may represent natural variations in physiological responses between animals, or the given state of light adaptation. It is also conceivable that the observed variations are representative of some higher order influence on the conductance properties of these neurons. Indeed there is some evidence for this in the L-neurons of the bee, as
Milde (1981) has shown that neurons of the same type may show a variety of markedly different responses to the same stimulus, and that a single unit is capable of switching between response types with no obvious change in external environment.

Specific characteristics of MD While the factors contributing to the step response are rather complex in all ocellar second-order neurons (for review see Laughlin 1981), this is especially true of MD. Descending Lneurons projecting from the median ocellus directly into the ventral nerve cord have been identified only in the bee (Pan and Goodman 1977) and caddisflies (Hagberg and Nässel 1986): they appear to be absent in other insects. Given the much larger transmission length in MD compared to the other L-neurons in the median ocellus, it is perhaps not surprising that this neuron also generates action potentials. Indeed, Milde and Homberg (1984) found that $\mathrm{L}_{\mathrm{D}}$ neurons (L-neurons descending into the ventral nerve cord) in the bee always generate spikes, often in combination with a graded response, but never show a pure graded response alone. Milde and Homberg (1984) also assert that only neurons showing spiking responses may respond to stimulation from sensory organs other than the ocelli. The $\mathrm{L}_{\mathrm{D}}$ neuron LMocth1, for example, responds to stimuli as diverse as wing motion, air streams over the head or abdomen, tactile stimulation, compound eye stimulation and the application of sugar solution to the antennae. In the present study we found that the spiking frequency of MD could be affected by manipulation of the wings and air streams blown over the head. Modulation of spike frequency by input from the compound eyes was not tested here, but appears likely on the basis of Kondo's (1978) descriptions from extracellular recordings of what would appear to be an MD equivalent in the dragonfly lateral ocellus. He found that at least one large efferent component is present in the nerve, which, in addition to responding to ocellar stimulation, also responds to wing beat and compound eye stimulation. Olberg (1981) also describes one of the descending self-movement detector neurons (BID) in the ventral nerve cord of the dragonfly as receiving ocellar input. This neuron receives its main input from several different sensory systems including the compound eyes, wind sensitive hairs on the neck and head, and neck proprioreceptors. The receptive fields and directionality of response of BID in different modalities are consistently aligned such that this neuron will optimally detect downwards shifts in pitch and contralateral shifts in yaw. Given the morphological shape and multimodal nature of MD it 
is a promising candidate for interaction in the selfmovement detection system. Alternatively, Milde and Homberg (1984) suggest that the presence of both graded and spiking modes of transmission may allow multiple channels of output from a single cell. In this case input from the ocelli is encoded by graded potential changes, while input from other sensory systems results in a modulation of spiking frequency. Feedback from other sensory systems to the ocelli may also act to 'gate' ocellar function such that visual input is synchronised with, for example, phases of wing motion.

\section{Preservation of spatial information}

Wiener kernel analysis of recordings from L-neurons stimulated by LEDs of randomly modulated intensity yields the first evidence for the preservation of spatial information at the level of the second-order neurons of any ocellus. Tasks involving spatial resolution are not generally considered to be a possible function of ocelli due to the presence of poorly focussed dioptrics, the irregular arrangement of rhabdoms and tapetum and high rates of convergence from photoreceptors to second-order neurons (Goodman 1981). Receptive fields of L-neurons have previously only been measured by Wilson (1978) along a horizontal trajectory in locust lateral ocelli. He found that field widths measured at $50 \%$ of maximum sensitivity were never less than $60^{\circ}$, and total extents were $130^{\circ}$, suggesting that resolving power in these neurons is lacking. However, our recent anatomical work (Stange et al. 2002) has demonstrated that a degree of spatial resolution may be one function of the median ocellus of dragonflies. Most notably the thickness and curvature of the lens is sufficient to form an astigmatic image focussed near the proximal limit of the retina. Additionally the photoreceptors are partially optically isolated by tapetal sheathing, an arrangement that has been shown to be beneficial both in terms of light capture and resolution for poorly focussed eyes of low $F$-number (Warrant and McIntyre 1991). Both adaptations suggest that spatial resolution may be present in the dragonfly median ocellus, with indications that resolution is higher in the vertical plane, and this was further supported by analysis of tapetal eyeshine (Stange et al. 2002). Recently, van Kleef et al. (2005) provide more direct evidence for the preservation of spatial information in the dragonfly median ocellus by measuring acceptance angles of individual photoreceptors in both the horizontal and vertical planes. They find the photoreceptors to have mean acceptance angles of $15^{\circ}$ in elevation and $28^{\circ}$ in azimuth, which supports the hypothesis of enhanced resolution in the vertical plane.
Here we provide evidence that spatial resolution is conserved after convergence of the photoreceptors onto the second-order neurons. For the purpose of illustrating essential features of the spatial map formed by the L-neurons, Fig. 8 provides a graphical representation of the data in Table 1 , showing average $50 \%$ of maximum sensitivity contours simplified to ellipses, and with positions relative to the overall field of view of the median ocellus. We find that centre points of all receptive fields lie close to, but consistently above, the equator of the eye. In azimuth, the total range covered is close to that of the total field of view, but in elevation the range is markedly narrower, with some acceptance angles approaching the $15^{\circ}$ lower limit imposed by the acceptance angles of individual photoreceptors (van Kleef et al. 2005). Furthermore, the widths and positions of the receptive fields differ considerably between L-neurons in a manner consistent with observation of their dendritic arborizations in the horizontal plane. There is a strong overlap within the azimuthal range covered, with some points being sampled by as many as six neurons.

The relationship between the extent of dendritic branching and receptive field in the vertical plane is less clear. From three-dimensional reconstructions of the L-neurons and from virtual projections of the brain in the frontal plane it appears that all L-neurons cover almost the entire range of the synaptic plexus in the vertical direction (Figs. 3b, 4d), and yet acceptance angles in elevation lie well below the limits imposed by the total field of view of the ocellus. The reasons for

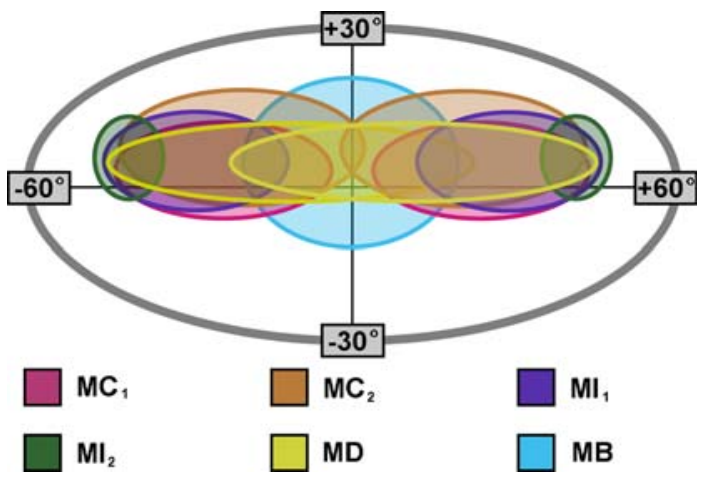

Fig. 8 Schematic illustration of the receptive fields of all Lneurons in the median ocellus. Receptive fields are simplified to ellipses with values for the major and minor axes defined by mean horizontal and vertical acceptance angles respectively. The origins of the ellipses are defined as the mean centre points of each neuron. The outermost ellipse describes the angular range over which tapetal reflections are visible from the ocellus (Stange et al. 2002). Note that as in Table 1, both centre points and acceptance angles of $\mathrm{MI}_{2}$ are underestimated as the receptive field of this neuron frequently exceeded the limits of the stimulus. 
this are currently unknown, however, several possibilities are worth considering. Firstly, from analysis of the figures given in Stange et al. (2002) it is apparent that the arrangement of photoreceptors in the vertical plane is not uniform. Receptors near the ventral and dorsal extremes of the retina are much shorter and of smaller diameter than those near the centre of the retina. It is therefore possible that the outputs of the retinal layer are weighted in the sense that photoreceptors near the centre of the retina will benefit from receiving more light, due to a larger rhabdom diameter, and will also be more efficient at absorbing incident light, due to the increased length of the rhabdom. Secondly, although the L-neurons are known to branch to a wide vertical area of the plexus nothing is known about the nature of the density of connections between first and second-order neurons. The output from the retinal layer may also be weighted in the sense that photoreceptors near the centre of the retina make more numerous and repeated synapses onto the second-order neurons. Thirdly, a pupil mechanism is present in the dragonfly ocelli such that during light adaptation a screening pigment migrates in the ventral to dorsal direction between the lens and retina. Stavenga et al. (1979) note that the pigment covers only the ventral part of the ocellus and therefore restricts light flux only from high elevations. As receptive fields were obtained from light adapted preparations it is likely that the vertical extent of the receptive field is partially determined by pigment migration. This would not, however, explain the restricted vertical receptive field at low elevations. Lastly, the $50 \%$ of maximum response criterion as used here does not fully account for the properties of the receptive fields: the spatial rolloff beyond this limit can be quite shallow, and we have also observed changes in receptive field shape over time which will be reported in a separate communication.

Overall, the median ocellar L-neurons appear to constitute an assembly of 11 sensors that together resolve some spatial detail in azimuth but not in elevation and can therefore essentially be regarded as forming a one-dimensional image of the equator over a wide azimuthal area (Fig. 8). In level flight, the optical axis of the median ocellus is nearly centred on the horizon which invites the conclusion that the system forms an internal representation or template of the horizon.

The presence of multiple sensors in the horizontal plane may have several benefits in terms of stability control. Different regions of visual space along the equator carry different information about movement in the natural world. Changes in pitch, for example, are optimally detected at an azimuth of zero, while changes in roll are optimally detected at azimuths of $90^{\circ}$ (Neumann and Bülthoff 2002). It is therefore conceivable that different L-neurons encode different information about in-flight attitude. Neurons such as $\mathrm{MB}$ and MD, which sample the medial region of visual space, may signal alterations in pitch, while the lateral neuron $\mathrm{MI}_{2}$ may signal changes in roll. Neumann and Bülthoff (2002) also note that the optimal receptive field for the detection of yaw rotations during forward flight is a narrow horizontal streak centred on the horizon. The one-dimensional arrangement of L-neuron receptive fields is reminiscent of an optimal yaw detector, although the large azimuthal acceptance angles of these neurons would restrict the detection of apparent yaw motion to all but the largest objects. Alternatively, if we assume that the function of the median ocellus is restricted to pitch control, then the presence of horizontally arranged sampling stations may provide an averaging function that enables the system to override local irregularities. If one or a few neurons are affected by some local feature, for example, the presence of the sun, then the majority of neurons which are not affected may average out or suppress the minority signal.

As the horizon itself is the most unambiguous cue for the determination of in-flight attitude, it was initially proposed that a degree of spatial resolution, especially in the vertical plane, might be utilised by the dragonfly median ocellus. Such imaging ability would allow the line of demarcation between the sky and ground to be used as an absolute reference point for the correction of pitch. A prerequisite for such corrective behavioural responses is the ability to perceive motion. Some evidence for motion selectivity in dragonfly ocelli has been provided by Zenkin and Pigarev (1971), who found motion selective responses in recordings from the ventral nerve cord of the dragonfly. Responses were largest to vertical motion of horizontal gratings and markedly smaller to horizontal motion of vertical gratings and could be attributed to the ocelli alone as they disappeared on occlusion of the ocelli, but not the compound eyes. However, the finding here that L-neurons are staggered in azimuthal, but not elevational space, would severely limit such a hypothesis in one respect, namely that this arrangement would only be useful for pitch correction when the dragonfly is already oriented close to the horizon. Large deviations in pitch attitude would render the horizon out of the field of view of all but the most lateral L-neurons. It is possible that in such a case the majority of L-neurons function as simple light sensors and monitor only 
temporal changes in illumination while reserving the role of fine pitch detectors for cases where body attitude is almost centered and fine corrective control is required, for example, during hover. Possibly some S-neurons, the receptive fields of which have not been determined for any insect, may act to roughly stabilise pitch deviations in cases where the horizon lies outside the field of view of the L-neuron.

An alternative hypothesis, which is not necessarily dependent on motion detection, is for a role in object avoidance. The sudden looming of a dark object, for example a tree trunk or an incoming predator, would cause large responses in only those neurons sampling that area of visual space such that corrective responses could be made to steer away from the oncoming object. In this case the one-dimensional imaging arrangement of the L-neurons is useful at any attitude in external coordinates as long as the animal flies forward relative to its own coordinate system. In fact, during forward translation only objects along a small elevation range around the equator are of interest as objects outside this range are not on a collision course. The receptive fields of some of the unilateral L-neurons described above bear similarities to the optimal receptive flow fields for collision detectors described by Neumann and Bülthoff (2002), which are elliptical with the long axis horizontal, and centred slightly above the horizon. The bilateral nature of the median ocellus in all insects is also intriguing in this respect. The dendritic branching of the majority of L-neurons in locusts is often limited to one lobe of the ocellar plexus (Taylor 1981). If some degree of spatial resolution in azimuth is permitted by the lens and retinal arrangement then this may also allow for a simple form of object avoidance. Simmons (1982a), however, finds that the dendritic arborizations in the lateral ocelli of locusts are often restricted to relatively small regions along the dorsoventral axis, suggesting that the function of the lateral ocelli may differ significantly from those of the median ocellus.

Lastly, we found that effects of stimulation with green light are only observable in the absence of a concurrent UV stimulus. This is entirely consistent with the reverse Purkinje shift known to occur in dragonfly ocelli whereby the system is more sensitive to UV at high illumination levels and to green at low illumination levels (Chappell and DeVoe 1975; Mobbs et al. 1981; Stange 1981). These results suggest that at the light levels used here, which are equivalent to bright daylight, the sensitivity of L-neurons to UV light far exceeds that to green light and that the latter is utilised only in lower light conditions, for example at dusk (Wilson 1978; Stange 1981).

\section{Conclusion}

We have identified what we believe to be a complete set of median ocellar L-neurons by a combination of intracellular staining and a novel method of tracing autofluorescent footprints of axon outlines. A hitherto unknown descending L-neuron has been described. Both the anatomy and physiology of this neuron are unique relative to other dragonfly L-neurons, but bear similarities to L-neurons described in other insects.

Our observations support a basic conclusion drawn from earlier observations on the optics and anatomy of the dragonfly median ocellus (Stange et al. 2002) and on the spatiotemporal transfer functions of its receptor neurons (van Kleef 2005), namely that this eye is capable of image vision. On the other hand, the functional significance of the observed type of mapping is by no means obvious. For a further understanding it will be necessary to augment the present observations by an investigation of the temporal dynamics of the median ocellar L-neurons and by an investigation of the properties of the lateral ocelli. Beyond this, it will be necessary to explore the way in which the outputs of the ocellar neurons project onto descending interneurons, as well as the extent to which they are integrated with other sensory modalities such as compound eye vision and mechanoreceptors, eventually contributing to the control of coordinated behaviour.

Acknowledgments This work was sponsored by the Air Force Office of Scientific Research (AFOSR), contract AOARD-034009. We thank Dr. Ulrike Schröter for her assistance with the three-dimensional reconstructions and Dr. Michael Ibbotson for providing the necessary software.

\section{References}

Chappell RL, DeVoe RD (1975) Action spectra and chromatic mechanisms of cells in the median ocelli of dragonflies. J Gen Physiol 65:399-419

Chappell RL, Dowling JE (1972) Neural organization of the median ocellus of the dragonfly. I. Intracellular electrical activity. J Gen Physiol 60:121-147

Chappell RL, Goodman LJ, Kirkham JB (1978) Lateral ocellar nerve projections in the dragonfly brain. Cell Tissue Res 190:99-114

Cornwell PB (1955) The functions of the ocelli of Calliphora (Diptera) and Locusta (Orthoptera). J Exp Biol 32:217237

Dowling JE, Chappell RL (1972) Neural organization of the median ocellus of the dragonfly. II. Synaptic structure. J Gen Physiol 60:148-165

Goodman C (1974) Anatomy of locust ocellar interneurons: constancy and variability. J Comp Physiol A 95:185-201

Goodman C (1976) Anatomy of the ocellar interneurons of acridid grasshoppers. I. The large interneurons. Cell Tissue Res 175:183-202 
Goodman LJ (1981) Organisation and physiology of the insect dorsal ocellar system. In:Autrum $\mathrm{H}$ (ed) Handbook of sensory physiology, Vol. VII 6C. Springer, Berlin Heidelberg New York, pp 201-286

Guy RG, Goodman LJ, Mobbs PG (1979) Visual interneurons in the bee brain: Synaptic organisation and transmission by graded potentials. J Comp Physiol A 134:253-264

Hagberg M, Nässel DR (1986) Interneurons subserving ocelli in two species of trichopterous insects: morphology and central projections. Cell Tissue Res 245:197-205

Homann H (1924) Zum Problem der Ocellenfunktion bei den Insekten. Z Vergl Physiol 1:541-578

James AC, Ruseckaite R, Maddess T (2005) Effect of temporal sparseness and dichoptic presentation on multifocal visual evoked potentials. Vis Neur 22:45-54

Klingman A, Chappell RL (1978) Feedback synaptic interaction in the dragonfly ocellar retina. J Gen Physiol 60:157-175

Kondo H (1978) Efferent system of the lateral ocellus in the dragonfly: its relationships with the ocellar afferent units, the compound eyes, and the wing sensory system. J Comp Physiol A 125:341-349

Laughlin S (1981) Neural principles in the peripheral visual systems of invertebrates. In:Autrum $\mathrm{H}$ (ed) Handbook of sensory physiology, Vol. VII 6B. Springer, Berlin Heidelberg New York, pp 133-280

Lee YW, Schetzen M (1965) Measurement of Wiener kernels of a non-linear system by cross-correlation. Int $\mathrm{J}$ Control 2:237-254

Lindemann JP, Kern R, Michaelis C, Meyer P, van Hateren JH, Egelhaaf M (2003) Flimax, a novel stimulus device for panoramic and highspeed presentation of behaviourally generated optic flow. Vis Res 43(7):779-791

Marmarelis PZ, McCann GD (1973) Development and application of white noise modeling techniques for studies of insect visual nervous system. Kybernetik 12:88-101

Milde J (1981) Graded potentials and action potentials in the large ocellar interneurons of the bee. J Comp Physiol A 143:427-434

Milde J (1984) Ocellar interneurons in the honeybee: structure and signals of L-neurons. J Comp Physiol A 154:683-693

Milde J, Homberg U (1984) Ocellar interneurons in the honeybee: Characteristics of spiking L-neurons. J Comp Physiol A155:151-160

Mizunami M (1994) Functional diversity of neural organization in insect ocellar systems. Vis Res 35:443-452

Mobbs PG, Guy RG, Goodman LJ, Chappell RL (1981) Relative spectral sensitivity and reverse purkinje shift in identified Lneurons of the ocellar retina. J Comp Physiol A. 144:91-97

Neumann TR, Bülthoff HH (2002) Behaviour-oriented vision for biomimetic flight control. Proceedings of the EPSRC/ BBSRS international workshop on biologically inspired robotics 14-16:196-203
Olberg RM (1981) Parallel encoding of direction of wind, head, abdomen and visual pattern movement by single interneurons in the dragonfly. J Comp Physiol A 142:27-41

Pan KC, Goodman LJ (1977) Ocellar projections within the central nervous system of the worker honey bee, Apis mellifera. Cell Tissue Res 176:505-527

Patterson JA, Chappell RL (1980) Intracellular responses of procion filled cells and whole nerve cobalt impregnation in the dragonfly median ocellus. J Comp Physiol A 139:25-39

Parry DA (1947) The function of the insect ocellus. J Exp Biol 24:211-219

Ruck P (1958) A comparison of the electrical responses of compound eyes and dorsal ocelli in four insect species. J Insect Physiol 2:261-274

Sakai HM, Naka KI, Korenberg M (1988) White noise analysis in visual neuroscience. Vis Neur 1:287-296

Simmons PJ (1982a) Transmission mediated with and without spikes at connexions between large second-order neurones of locust ocelli. J Comp Physiol A 147:401-414

Simmons PJ (1982b) The operation of connections between photoreceptors and large 2nd order neurones in dragonfly ocelli. J Comp Physiol A 149:389-398

Stange G (1981) The ocellar component of flight equilibrium control in dragonflies. J Comp Physiol A 141:335-347

Stange G, Stowe S, Chahl JS, Massaro A (2002) Anistropic imaging in the dragonfly median ocellus: a matched filter for horizon detection. J Comp Physiol A 188:455-467

Stavenga DG, Bernard GD, Chappell RL, Wilson M (1979) Insect pupil mechanisms. III. On the pigment migration in dragonfly ocelli. J Comp Physiol A 129:199-205

Strausfeld NJ (1976) Atlas of an insect brain. Springer, Berlin Heidelberg New York

Taylor CP (1981) Graded interactions between identified neurons from the simple eyes of an insect. Brain Res 215:382387

Toh Y, Hiroshi S, Iwasaki M (1983) Ocellar system of the insect: comparison of dorsal ocellus and lateral ocellus. Vis Res 23:313-323

van Kleef J, James AC, Stange G (2005) A spatiotemporal white noise analysis of photoreceptor responses to UV and green light in the dragonfly median ocellus. J Gen Physiol 126:481497

Warrant EJ, McIntyre PD (1991) Strategies for retinal design in arthropod eyes of low F-number. J Comp Physiol A 168:499-512

Wehner R (1987) 'Matched filters'-neural models of the external world. J Comp Physiol A 161:511-531

Wilson M (1978) The functional organisation of locust ocelli. J Comp Physiol A 124:297-316

Zenkin GM, Pigarev IN (1971) Optically determined activity in the cervical nerve chain of the dragonfly. Biofizika 16:299306 